\title{
Physiological and pharmacological aids in the differential diagnosis of tremor ${ }^{1}$
}

\author{
BHAGWAN T. SHAHANI AND ROBERT R. YOUNG \\ From the Department of Neurology, Harvard Medical School, \\ and Laboratory of Clinical Neurophysiology, Massachusetts General Hospital, Boston 02114, USA
}

SYNOPSIS Physiological and pharmacological studies of more than 150 patients with movement disorders are reported. Particular attention is paid to the differentiation of various types of tremor on the basis of rate, rhythm, and pattern of EMG activity in antagonistic muscles. The typical 'tremor-at-rest' of Parkinson's disease-3-7 Hz activity which alternates between antagonistic muscles-is suppressed, at least briefly, during voluntary activity, at which time typical 8-12 $\mathrm{Hz}$ 'physiological tremor' may be seen. Essential tremor and its familial or senile variants also have a characteristic EMG pattern during voluntary activity $-5-8 \mathrm{~Hz}$ bursts of activity which are synchronous in antagonistic muscles. This type of tremor may also be present in patients with Parkinson's disease and in certain kinships with a Charcot-Marie-Tooth polyneuropathy. Other tremors in association with polyneuropathy ('neuropathic tremor') have different physiological characteristics. Myoclonus is of essentially two types ('positive' with EMG bursts and 'negative' with brief pauses in ongoing activity, as with asterixis) and may, at times, mimic tremor. Certain specific tremors respond predictably to specific pharmacological therapy.

Tremor can be defined as a more or less regular, rhythmical oscillation of a body part about a fixed point or plane. It may be due to alternating or synchronous contraction of antagonistic muscle groups. Other abnormal movements, such as shivering, shuddering, and certain types of myoclonus, should probably be differentiated from tremor though, at times, it may be difficult to ascertain clinically whether these or other movements do, in fact, fulfil the stated criteria for tremor. Nevertheless, the present intuitive concept of tremor is useful until more precise clinical, physiological, or pharmacological definitions can be agreed upon.

Tremor is a very common clinical entity; indeed, a tremor is visible at some time in the outstretched hands of all normal persons. There are a number of different types of tremor, as will be outlined below, and they are often confused

\footnotetext{
1 Presented in brief at the VIIIth International Congress of Electroencephalography and Clinical Neurophysiology, Marseilles, France, September 1973.
}

(Accepted 3 April 1976.) with one another. Strict clinical criteria have been proposed to divide tremors into several categories, but it still may be difficult to differentiate among them by clinical observation. This review presents our experience with clinical, physiological, and pharmacological evaluation of a number of patients, particularly as the observations relate to several questions: (1) how can one differentiate, using physiological parameters, one tremor from another, (2) which pharmacological agents are useful in the treatment of which tremors, and (3) what do these observations imply about the pathophysiological mechanisms involved? A brief summary of these findings has been presented (Shahani and Young, 1973).

\section{METHODS}

The signs and symptoms of the more than 150 patients studied included: typical essential tremor, affecting primarily the upper limbs and fulfilling the criteria listed below; its familial and senile variants; the resting tremor of Parkinson's disease; patients 
with Parkinson's disease who have three different types of tremor and myoclonus; the 'cerebellar' tremor of Friedreich's ataxia; 'rubral tremor' from lesions in the superior cerebellar peduncle; asterixis, simulating a tremor in association with hepatic cirrhosis and hyperammonaemia, chronic pulmonary disease and hypercarbia, renal failure, or anticonvulsant therapy; post-hypoxic intention myoclonus; progressive myoclonic epilepsy; tremor in association with chronic, often relapsing and steroid-sensitive peripheral neuropathy; and Charcot-Marie-Tooth distal, primarily motor, neuropathy and coexisting essential tremor which has been confused with the Roussy-Lévy syndrome (Yudell et al., 1965). Several otherwise normal subjects were studied who demonstrated peculiar tremors in circumstances to be described below.

After historical aspects of these illnesses were noted, the tremors were studied clinically; they were graded on a 0 to $4+$ scale in each limb at rest and in various postures, including the use of drinking cups. Handwriting specimens were obtained, and the patients were often photographed with a $16 \mathrm{~mm}$ motion picture camera or recorded on video-tape. Tremor amplitude was documented by feeding the output of two Grass Model SPA-1 accelerometers (taped to the patient in planes at right angles to one another) into DC amplifiers, the outputs of which were displayed on an ink-writing polygraph. Areas of maximum tremor (fingers, forehead, jaw, or feet) were chosen for application of these transducers, and patients were usually also examined while seated, with both arms outstretched horizontally and the accelerometers on the fingers. The same experimental conditions, recording sites, and accelerometer positions were repeated in any one patient during each recording session. Recordings were also made during certain movements, such as finger-nose testing, speaking, and writing. In addition, surface, needle, or thin intramuscular wire EMG recordings were made from the appropriate muscles, using the ink-writing polygraph and/or an oscilloscope with either a kymograph or Polaroid camera. For tremor recording, antagonistic groups of muscles, such as extensors and flexors of the wrist or elbow, were usually chosen. These clinical, EMG, and accelerometer tests were carried out before and during specific pharmacological therapy for various tremors, as outlined below.

Direct recording of tremor amplitude-that is, the maximum displacement of the body part from one extreme, through the midpoint, to the opposite extreme-can be carried out only with considerable difficulty and usually requires one restraint or another upon free movement. However, when dealing with Simple Harmonic Motion, to which tremors (especially the almost completely sinusoidal physiological or essential tremors) are a close approximation, the acceleration of a moving point with reference to a fixed point equals a constant (k) times the displacement of the former point from the latter. The constant $(k)$ equals the square of the angular velocity of the moving point, the angular velocity being $2 \pi$ times the frequency of motion. Therefore, if the frequency of tremor in a limb of any one patient is constant from one recording to another, any change in tremor amplitude will be reflected as a directly proportional change in acceleration of the limb. For that reason, and because acceleration, tremor frequency, and rhythmicity are easily measured by accelerometers, they were used in these studies of tremor. There was no statistically significant change in tremor frequency in any one patient from one recording to another, and tremor amplitude could, therefore, be expressed as acceleration. Since, after each recording, the accelerometers were calibrated, using the acceleration caused by gravity $(g)$ by holding the recording axis vertical and rotating the accelerometer $180^{\circ}$ (producing a pen deflection of $2 g$ - that is, $-1 g$ to $+1 g$, or vice versa - the units are given throughout as multiples of $g, 1 g$ being $9.8 \mathrm{~m}$ per $\mathrm{s}^{2}$. The absolute displacement can, in any instance, be estimated by dividing the recorded acceleration by $(2 \pi \text { Freq })^{2}$.

\section{RESULTS}

In normal persons, a fine rapid tremor was usually seen when the upper extremities were outstretched. In some people it was coarser, while in others it was difficult to see at all without the aid of mechanical amplifying devices. Not only did the amplitude of this tremor vary from one person to the next, but it was also quite variable in the same individual from one time to another. If it did not exceed the threshold for production of symptoms and was rapid $(8-12 \mathrm{~Hz})$, this tremor was termed 'physiological'. EMG recordings from pairs of antagonistic muscle groups in most of these subjects were unremarkable and had the appearance of an interference pattern' (Fig. 1); in a few, discrete, synchronous bursts of EMG activity occurred. Physiological tremor was unaffected by the administration of propranolol or alcohol.

When the upper extremities were outstretched, the appearance of the movement disorder in patients with essential tremor (or its familial and senile variants) was clinically similar to that seen 

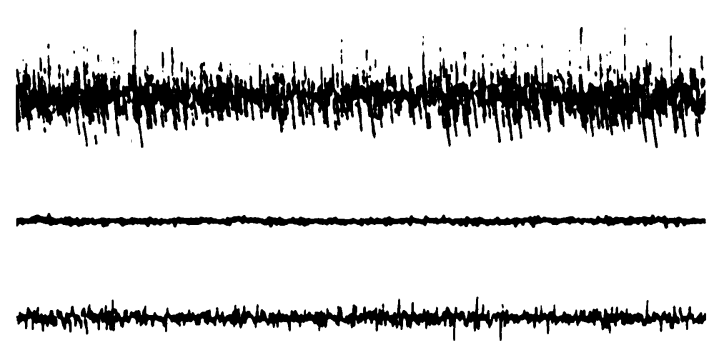

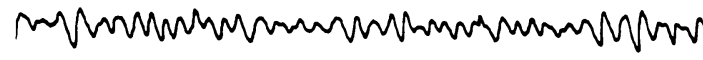

FIG. 1 Physiological tremor recorded from a normal subject. Upper four tracings are surface ink-written EMG activity from extensors and flexors of wrist and extensors and flexors of elbow (top to bottom tracing, respectively), all in the same arm. Accelerometric recording is shown in the lower tracing. Calibrations$1.0 \mathrm{~s}$ and $50 \mu \mathrm{V}$ or $1.0 \mathrm{~g}$.

with physiological tremor. Both these action tremors were rhythmical, and their mechanograms were regular and almost sinusoidal in nature. The symptomatic essential tremors, however, were of larger amplitude and slower rate $(4.25-8.25 \mathrm{~Hz}$ ) (Fig. 2). Simultaneous surface EMG recordings from extensors and flexors of the wrist and fingers characteristically showed bursts of EMG activity which occurred synchronously in these antagonistic muscle groups (Figs. 2, 3, and 4). In less than 5\% of the patients we have studied with essential, familial, or senile tremor, the EMG activity in these antagonistic muscles (or in tibialis anterior and triceps surae, when they were involved) was 'alternating', a feature characteristic of the 'tremor-at-rest' of Parkinson's disease. Nonetheless, these few patients had no clinical evidence of Parkinson's disease at that time. Moreover, they, like the other $95 \%$, had the following clinical features of a typical essential tremor. There was a long history of insidious onset and gradual progression. The tremor was absent at rest and clearly present, though submaximally, in the outstretched arms. It was accentuated when the limb was used for tasks requiring careful attention, such as writing and carrying a full cup. This was different from the 'cerebellar tremors' which were minimal with the arms outstretched and much more severe as part of the ataxia or dysmetria of finger-nose testing. Essential tremor, in most patients, was suppressed by (1) amounts of ethyl alcohol insufficient to produce lethargy or drowsiness and (2) the chronic oral administration of propranolol (60 to $240 \mathrm{mg}$ /day). The combination of diazepam and propranolol often produced striking relief when the latter alone was only moderately effective. Phenothiazines or haloperidol worsened essential tremor considerably. The physiological and pharmacological characteristics of essential tremor were identical with those of senile and familial tremor.

We had the opportunity of studying several patients with tremors that appeared to be on a voluntary basis. In these instances, the patients were very suggestible, and some were considered to be frankly hysterical. Their tremor could be started and stopped abruptly upon suitable request. The surface EMG from the affected muscles of some of those patients again showed simultaneous activity in extensors and flexors, indistinguishable from that seen in Fig. 4. Other such patients produced bursts of EMG activity primarily in the flexor group. Many normal subjects (Fig. 5) found it was possible, by tensing the appropriate muscles, to reproduce the tremor presented by these latter patients-namely, rapid, 9-10 Hz bursts of tightly grouped EMG activity in the flexors. A more detailed search might have revealed some rhythmical activity in the extensors, but it was certainly much less obvious than in the other patients we have studied. Slower 'voluntary' tremors, on the other hand, were produced by alternating activity in the antagonistic muscle groups.

In contrast with the simultaneous or 'synchronous' bursts of EMG activity seen with essential tremors, the activity recorded from the same antagonistic muscle groups during the tremor-at-rest of Parkinson's disease was of an alternating character, as seen in the top half of Fig. 6. The EMG activity in extensors alternated 


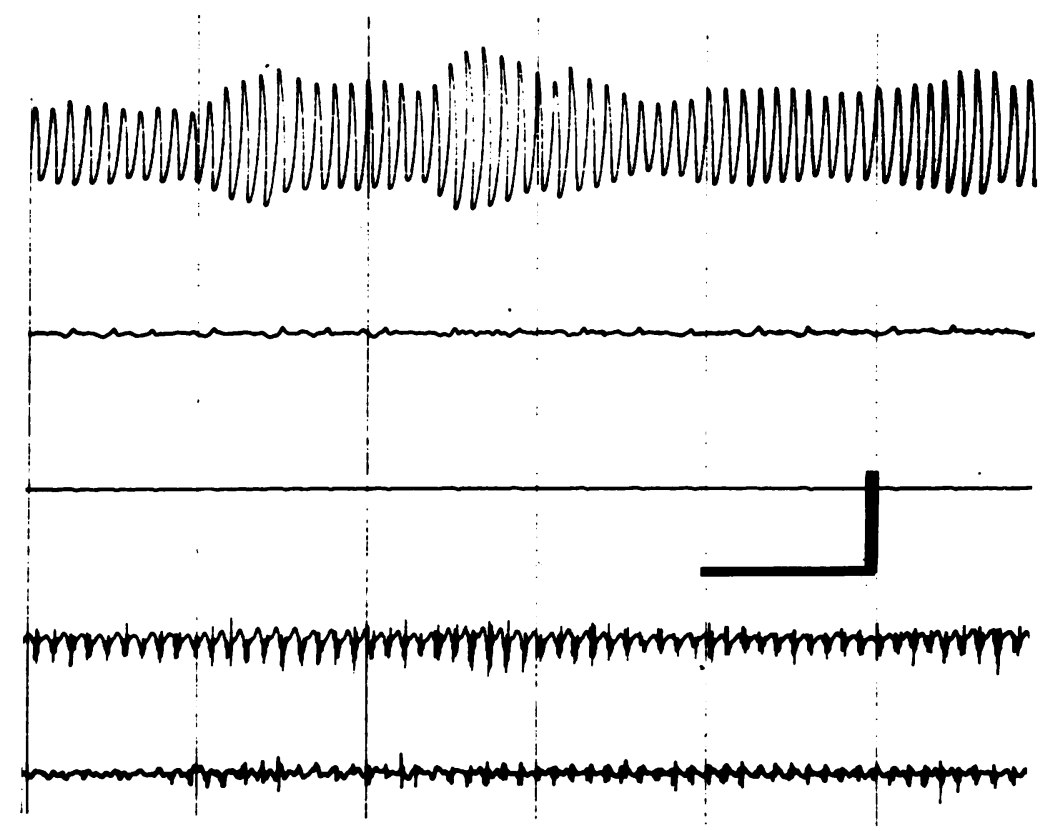

FIG. 2 Accelerometric recording (upper tracing) of tremor is combined with surface, ink-written EMG activity (bottom two tracings) recorded from extensors (above) and flexors (below), all of the same hand in a patient with essential tremor. Calibrations $-2 \mathrm{~s}$ and $50 \mu \mathrm{V}$ or $1.0 \mathrm{~g}$. Note the simultaneous EMG activity in the antagonist muscles, together with the sinusoidal movement of the tremor.
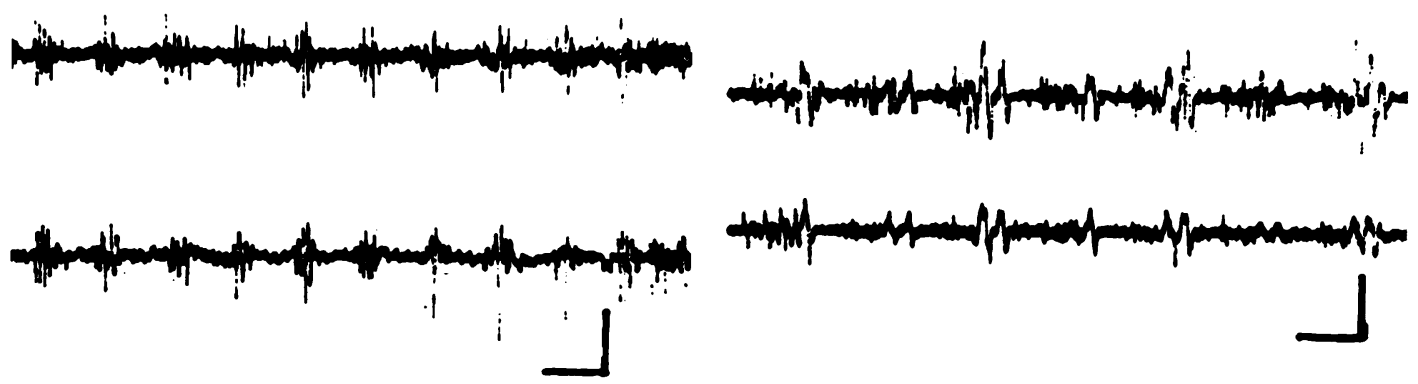

FIGS 3 and 4 Surface EMG activity is recorded in the upper tracing, in each case from the wrist and finger extensors and in the lower from the flexors of the wrist and fingers in the forearm. Calibration-vertical: $200 \mu \mathrm{V}$; horizontal: $200 \mathrm{~ms}$ in Fig. 3 and $100 \mathrm{~ms}$ in Fig. 4. The tremor bursts are quite regular in Fig. 3 and less so in Fig. 4, but in both patients with essential tremor, the bursts are seen to be simultaneous in the two antagonist muscle groups. 


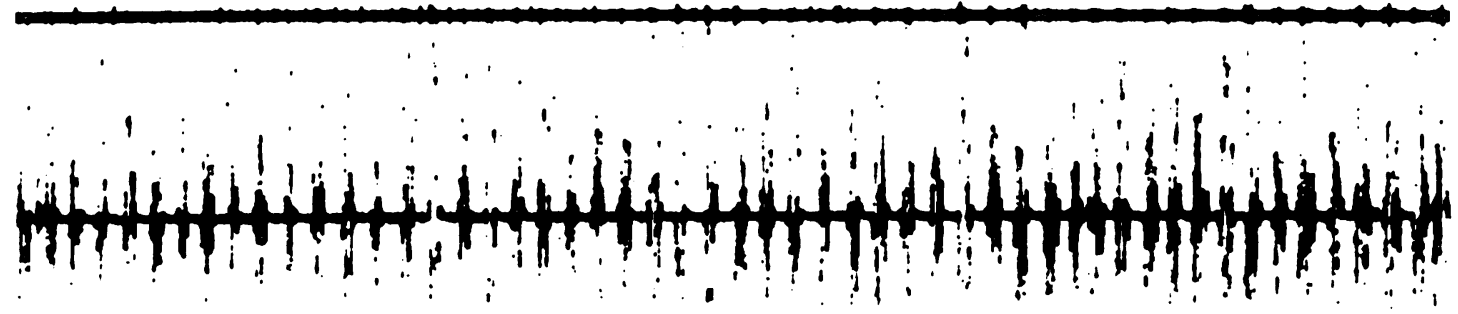

FIG. 5 Surface EMG activity from extensor (above) and flexor (below) muscle groups of the ankle in a normal subject who has learned to produce a 'voluntary tremor'. No clear activity is recorded from the extensors-that which is seen on the upper trace appears to be recorded by volume conduction from the flexors. The calibration represents $1.0 \mathrm{~s}$.
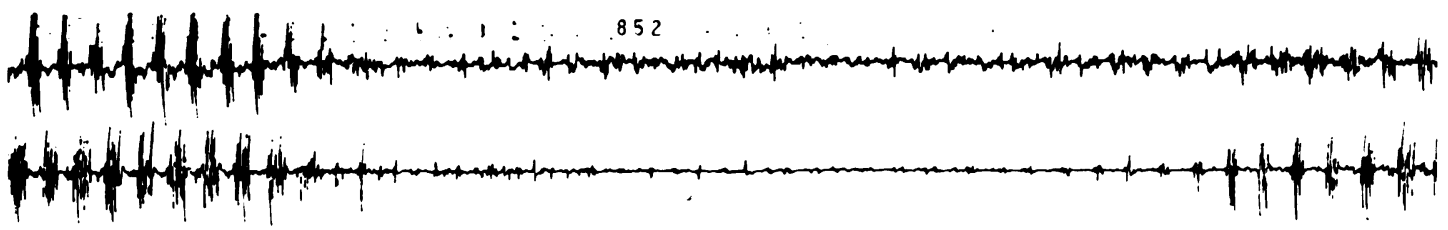

1

Whivitum

FIG. 6 Accelerometric recording (lower tracing) of the tremor-at-rest with surface, ink-written EMG activity (upper two tracings) recorded from extensors (above) and flexors (below) all of the same hand in a patient with Parkinson's disease. Note that, with attempted voluntary effort at the arrow, the typical alternating EMG activity and accelerometric tracing of tremor almost disappear. Calibrations-1.0 s and $50 \mu \mathrm{V}$ or $1.0 \mathrm{~g}$.

with that in flexors as the finger or hand moved up and down to produce the rhythmical tremorat-rest depicted at the beginning and end of the bottom line of Fig. 6. In patients with Parkinson's disease, this tremor was present with the limbs more or less in repose, was suppressed (at least briefly) during voluntary activity (see arrow, Fig. 6), and was produced characteristically by alternating 3-7 Hz contractions of antagonistic muscle groups, features quite opposite to those seen with essential tremor. Physiological tremor was also present in patients with Parkinson's disease and was accentuated by certain movements. A few patients (approximately 5 to $10 \%$ of those with Parkinson's disease) had, in addition, typical essential tremor. In those patients in whom both tremor-at-rest and an action tremor were present, one witnessed, as in Fig. 7, a shift from the alternating bursts in extensors and flexors at rest to sustained activity 


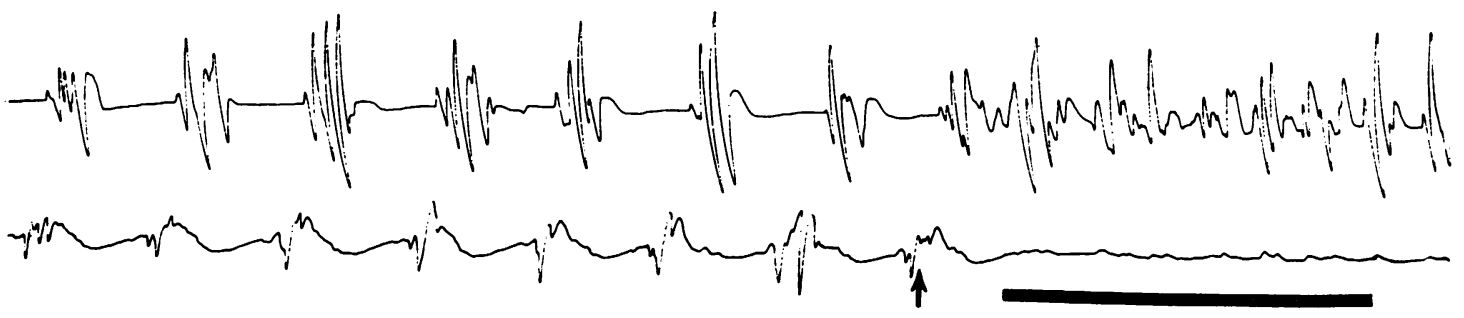

FIG. 7 An ink-written EMG recording from extensor (above) and plantar flexor (below) muscle groups in the leg of a patient with a typical tremor-at-rest which can be seen as the alternating bursts to the left of the arrow. At the arrow, the patient begins voluntarily to contract his dorsiflexors of the foot and the slower alternating activity is replaced by continuous, albeit grouped and tremulous, activity in these muscles. This is the counterpart of his faster 'action tremor'. Calibration is $1.0 \mathrm{~s}$.

in the active muscle group when contraction of it was voluntarily performed. During this contraction, a tendency for grouping of EMG bursts into an action tremor was seen, albeit at a faster frequency than the bursts seen during the resting tremor. In many instances, a tremor with all the characteristics of the tremor-at-rest reappeared despite the continued contractions of these muscles. Ethopropazine was found to be the most useful of the standard therapies for Parkinson's disease as far as resting tremor was concerned, though L-dopa also diminished this tremor in some patients. In other patients, L-dopa had worsened the coexisting essential tremor considerably, even at relatively low dosages. By the combined administration of propranolol and L-dopa, it was possible to reach levels of L-dopa that were sufficient to benefit most of these patients without their being limited by the action tremor. Phenothiazines and haloperidol worsened the tremor-at-rest, as well as any coexistent essential tremor.

During the systemic administration of isoproterenol (the $\beta$-adrenergic stimulating agent) or under conditions of stress or anxiety, a higher amplitude tremor was recorded from normal subjects or patients with the tremors described above. The frequency of this 'new tremor' was unchanged from that of the pre-existing physiological, essential, or resting tremor. In subjects with the former tremor, the presence of discrete, synchronous EMG bursts was more evident. During these conditions, the intravenous administration of $\beta$-adrenergic receptor blocking doses of propranolol (2 to $5 \mathrm{mg}$ ) acutely eliminated the exaggerated response but did not reduce the underlying tremor, whatever type it was.

In six patients with biopsy-proven, chronic relapsing, usually steroid sensitive polyneuropathy, a prominent tremor of the action type appeared at one phase of their disease. Though it, too, was most obvious distally, was present with the arms outstretched, continued throughout movement but was exaggerated by certain movements or postures, and disappeared immediately when the limbs were relaxed, it was not affected by propranolol. Comparable EMG recordings from these patients with 'neuropathic tremor' showed an intermediate situation. That is (1) the bursts of EMG activity were less well grouped than in the synchronous or alternating varieties of tremor and (2) they, at times, appeared to be synchronous in antagonistic muscles and, at other times, alternating. This may be seen in Fig. 8, taken from a patient with neuropathic tremor. Note that the grouping of muscle action potentials was irregular and poorly defined, and the accompanying movement, as depicted in the accelerometric record, was correspondingly less regular or sinusoidal than the tremors described previously.

Another, more common, clinical setting with prominent irregular contraction of skeletal muscle was asterixis, in which brief nonrhythmical pauses of varying lengths, during voluntary muscle contraction, were present. This produced an unstable, tremulous posture which often masqueraded as tremor, though it had its own clinical setting and typical EMG picture. In 


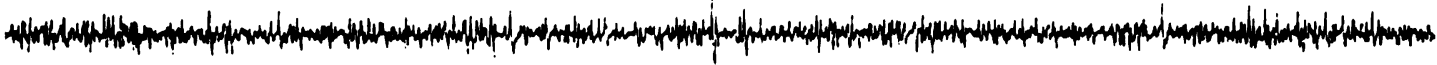

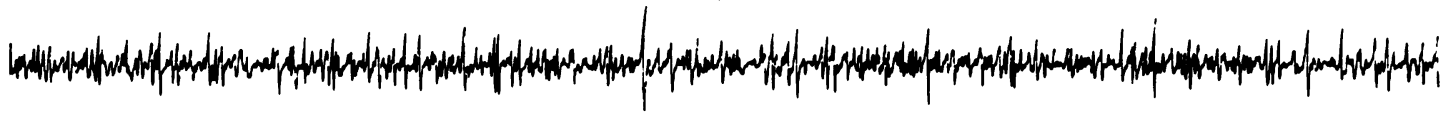

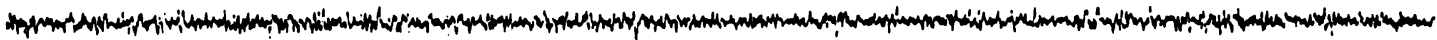

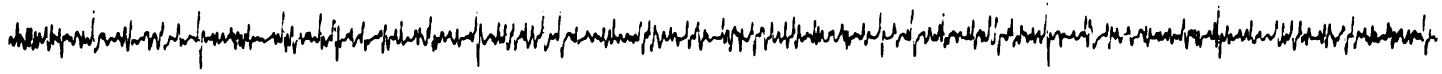

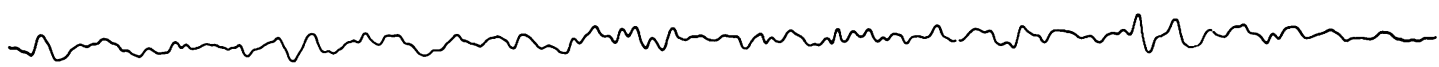

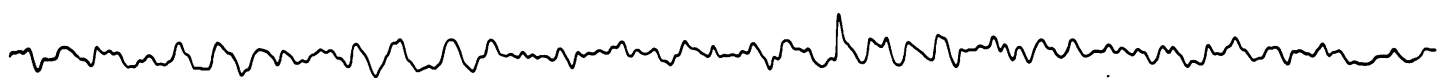

FIG. 8 Accelerometric recording (lower two tracings) of 'neuropathic tremor' with surface ink-written EMG activity from extensors and flexors of wrist (upper two tracings) and extensors and flexors of elbow (middle two tracings), all recorded from the same arm. Note the irregular grouping of action potentials and the lack of clear-cut correlation between the activity in the two antagonist groups. Calibrations-1.0 s and $50 \mu \mathrm{V}$ or $1.0 \mathrm{~g}$.

Fig. 9, the irregular contraction of antagonistic muscles may be seen on the top two lines, and the fluctuating movement is recorded accelerometrically at the bottom. Longer synchronous pauses in contraction, such as at the arrows, were associated with grosser movements (which clinically are termed 'flap' or 'asterixis') with larger acceleration and amplitude. A similar situation in a different patient in Fig. 10, where the rather smooth voluntary contraction of wrist extensors was interrupted above the arrow by a pause in the on-going EMG activity, was associated with a striking change in acceleration (and displacement) of the hand, particularly with resumption of normal contraction, and was followed by shorter pauses and a correspondingly tremulous accelerometric record. It was sometimes difficult to be certain from a short stretch of EMG record whether one was dealing with asterixis or a poorly synchronized essential or neuropathic tremor. Further recordings, however, showed the longer synchronous pauses and longer periods of contraction without pause typical of asterixis. The lapses of posture during the brief pauses in the voluntary contraction, or the recovery therefrom, once the pause was over, produced the quick, lightning-like movements which, to the eye, appear typical of myoclonus. In addition to these patients with asterixis, there were patients with myoclonus in whom the quick movements consisted of brief, irregular contractions, ranging from single motor units to groups of units which occurred either at rest (Fig. 11) or were superimposed upon a continuous background contraction. Propranolol did not affect the tremulousness associated with asterixis or post-hypoxic intention myoclonus.

In patients with Friedreich's ataxia, the 'tremor' was minimal with the hands outstretched and much more severe as the hand approached 

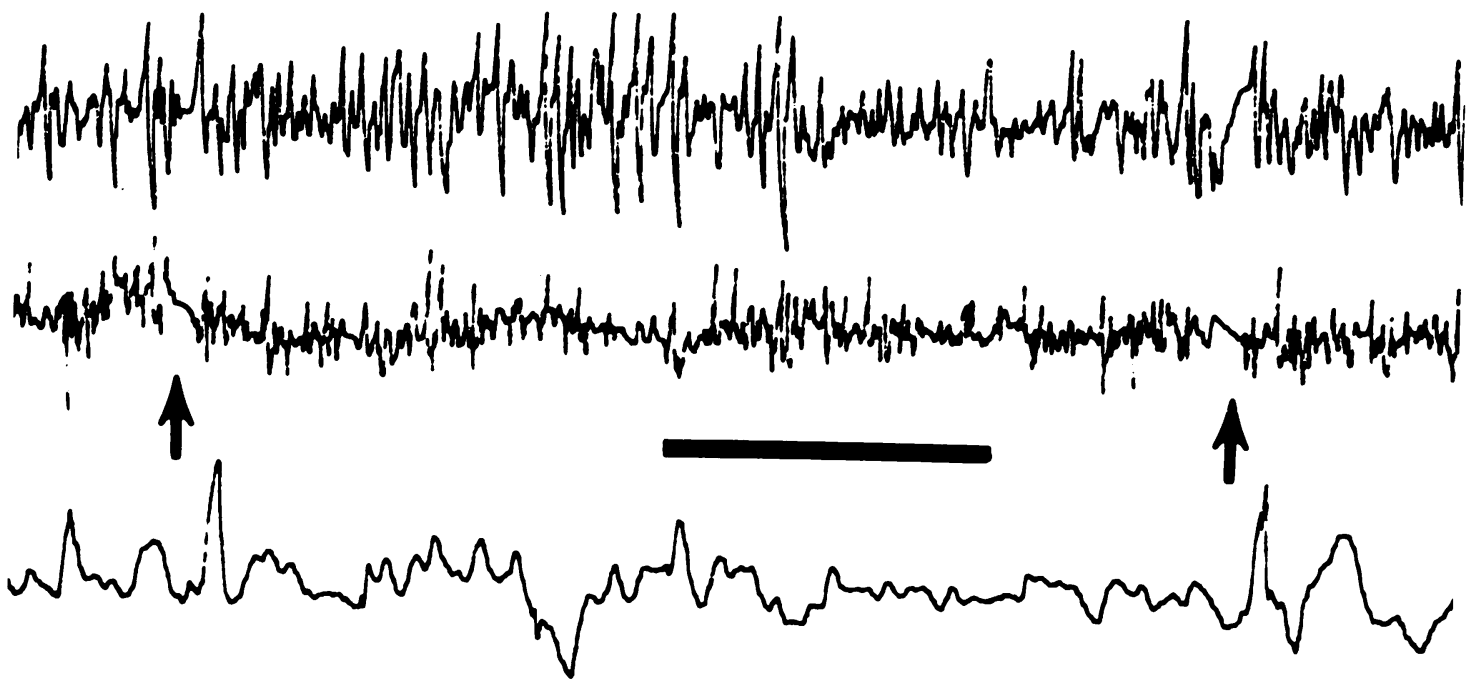

FIG. 9 An ink tracing of EMG activity in a patient with asterixis from extensors (top) and flexors (middle) of the wrist and fingers, associated with voluntary maintenance of posture (hand outstretched). The bottom tracing is from an accelerometer attached to the hand. Calibration is $0.5 \mathrm{~s}$. Note the irregular EMG activity with tremor being recorded on the mechanogram. Longer pauses in EMG activity in both muscle groups, as at arrows, are associated with movements of larger amplitude.
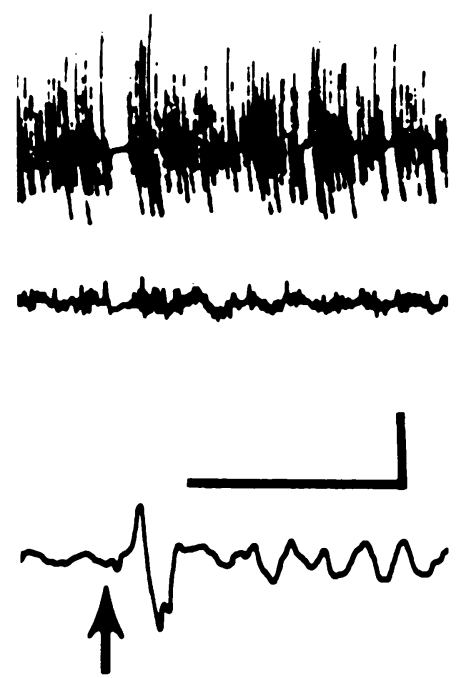

FIG. 10 Figure 10 is similar to Fig. 9. The activity in extensors and flexors of wrist (upper two tracings) is interrupted by a period of EMG silence (arrow) resulting in gross movement signalled by the accelerometer (lower channel). An almost regular tremor is seen thereafter, associated with the briefer pauses. Calibration is $0.5 \mathrm{~s}$ and $50 \mu \mathrm{V}$ or $1.0 \mathrm{~g}$. a target, such as on finger-nose testing. In contrast with essential tremor, which was usually in one plane, the direction of the abnormal movement during the 'cerebellar tremor' was multi-planar and the large oscillations highly irregular and slow ( 2 to $4 \mathrm{~Hz}$ ). Though the cerebellar tremors were usually absent with the limbs in repose, 'rubral tremors' were often present under those conditions though they increased considerably with activity. Propranolol had no effect on these 'cerebellar tremors', and alcohol usually worsened them.

\section{DISCUSSION}

Although the EMG recordings do provide some little insight into the mechanisms of tremor, they are primarily useful for several other reasons. First, they allow one to be certain that, in studying these tremors, he is dealing with active muscle contraction and not passive movement, as is the case with ballistocardiographic artefact, which appears to account for most of the tremor at rest seen in normal circumstances (Brumlik 


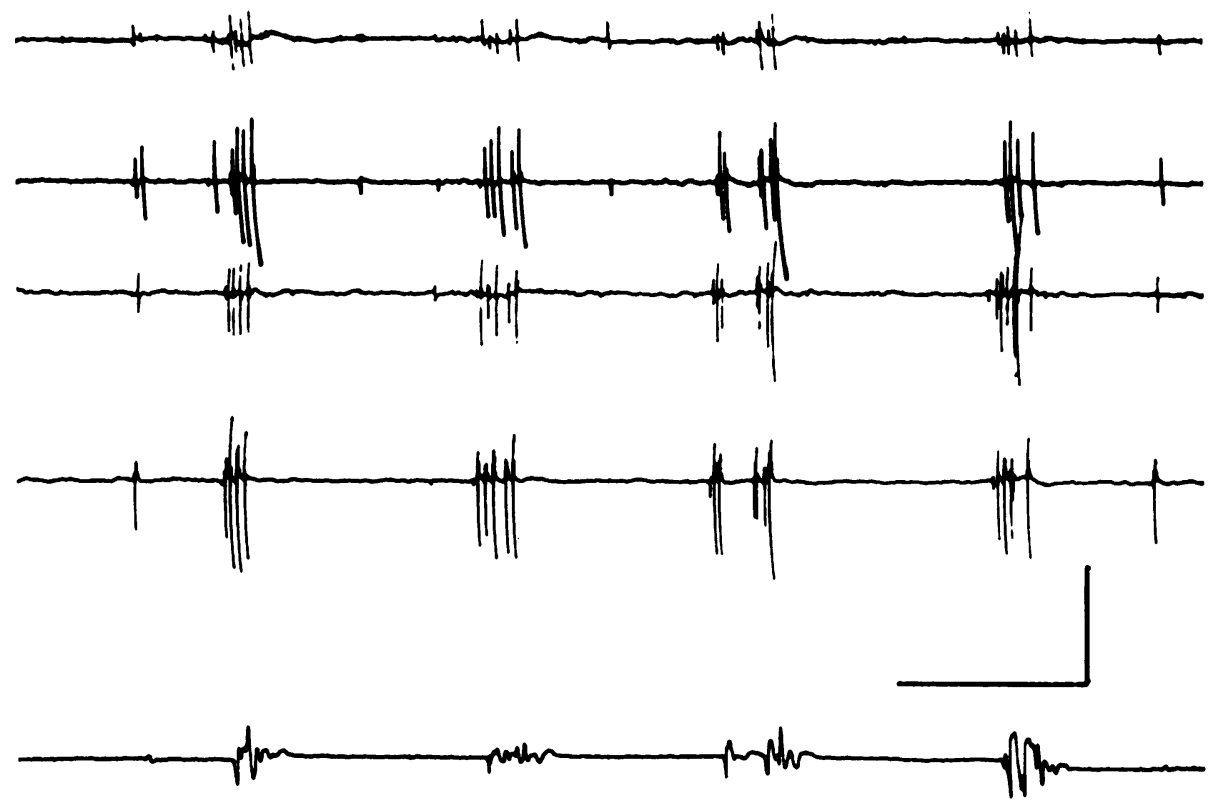

FIG. 11 Accelerometric recording (lower tracing) and surface EMG recording (upper four traces) from extensors and flexors of wrist and extensors and flexors of elbow (from top to bottom, respectively) in a patient with progressive myoclonic epilepsy. Note the synchronous bursts of EMG activity. Calibrations-1.0 s and $50 \mu \mathrm{V}$ or $1.0 \mathrm{~g}$.

and Yap, 1970). Second, the EMG observations caution against definitions of tremor which include statements about 'alternating muscle contraction'. In Table 1 of Brumlik and Yap (1970), nine of the 15 definitions of tremor from various authorities include reference to contraction, usually alternate, of opposing muscle groups (also see Hardy et al., 1970; Fahn, 1972). As long ago as 1940, Hoefer and Putnam described synchronous contraction of antagonistic muscles in abnormal tremor, and Lippold ct al. (1957) clearly report it as the basis in man of what they termed physiological tremor. One readily appreciates that alternating contraction of antagonist muscles would, if the frequency were high enough, produce tremor, but the notion that synchronous bursts of activity can also do so is not an intuitive one. Presumably, inequalities in strength and timing of contraction account for the tremor. When one considers the 'tremor' of myoclonus or asterixis (Adams and Foley, 1953), it is also easy to visualize irregular increases or pauses in contraction producing oscillations of the limb (Leavitt and Tyler, 1964). EMG recordings also stress that the quick, lightning-like, jerky movements typical of clinical myoclonus are caused, in patients with posthypoxic intention myoclonus as well as those with asterixis, by (1) lapses of posture during the brief pauses in voluntary contraction or (2) the instantaneous recovery therefrom when the EMG returns after the pause is over. Because these synchronous brief pauses, which occur at irregular intervals in the ongoing voluntary EMG activity, produce movements that appear clinically to be myoclonic, one may characterize this as 'negative myoclonus'. These pauses in EMG activity presumably result from either inhibitory discharges or disfacilitation (cessation of excitatory activity) within the CNS and can be contrasted with the irregular brief bursts of EMG activity seen synchronously in many otherwise relaxed muscles of the same limb in patients with other types of visible myoclonus ('positive myoclonus'). The latter excessive paroxysmal excitation (at the lower motor 
neurone at any rate) is well recognized as the EMG counterpart of myoclonus but must be differentiated from the excessive paroxysmal 'inhibition' which produces clinically very similar myoclonic jerks.

The EMG recordings may assist in diagnosis as well, as illustrated in Fig. 12. As new rather

A
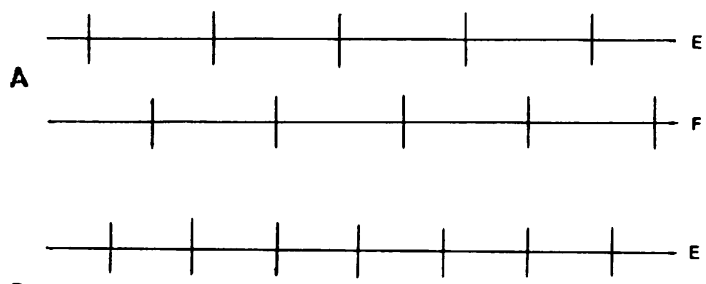

B
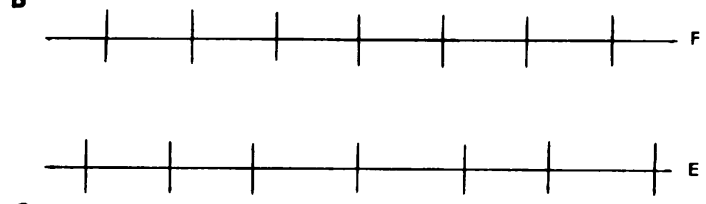

C
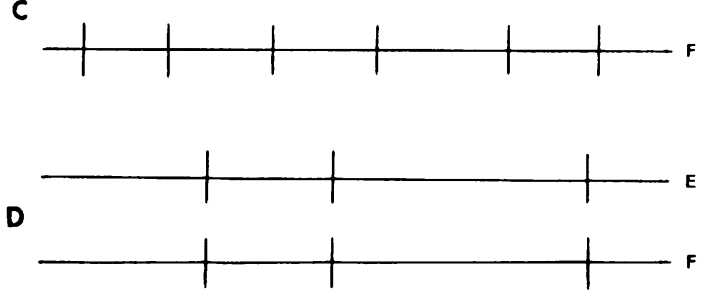

FIG. 12 Schematized drawing to represent the EMG bursts of activity in extensors $(E)$ and flexors $(F)$ in the forearm of patients with $(A)$ tremor-at-rest associated with Parkinson's disease, (B) essential tremor, $(C)$ neuropathic tremor, and (D) myoclonus. The baseline in each instance represents roughly $1.0 \mathrm{~s}$. See the text for details.

specific therapies appear, more accurate diagnosis becomes important so that, on the one hand, patients who are unlikely to benefit are not exposed unnecessarily to potentially toxic therapeutic agents and, on the other hand, effective therapies are not discredited by use in situations where they are not likely to be helpful. Most patients with tremors are not improved by propranolol, though the great majority of those with essential tremor are (Sevitt, 1971; Winkler and Young, 1971, 1974; Gilligan et al., 1972;
Pakkenberg, 1972; Dupont et al., 1973; Morgan et al., 1973; Tolosa and Lowenson, 1975). Critchley (1949) suggested that certain other neurological abnormalities, such as spasmodic torticollis or olivopontocerebellar degeneration, coexist with, and may be variants of, the essential tremor syndrome. If further therapeutic trials in these other conditions continue to show no effect, it may be useful to redefine the syndrome of essential tremor more strictly, with the inclusion of pharmacological criteria.

It has long been assumed that it is the amplitude of physiological or, to use Brumlik and Yap's terminology (1970), 'normal postural tremor' that is increased by thyroid hormone or adrenaline, endogenously released, as with relative hypoglycaemia or fright, or exogenously administered; by withdrawal from various CNS depressants - for example, alcohol or narcotics; by anxiety, the exact catecholamine and other substrates for which remain to be demonstrated; and by the administration of lithium in psychoactive doses. If it becomes noticeable or symptomatic, the 'abnormal postural tremor' is then designated according to the circumstances responsible for its exacerbation-for example, 'thyrotoxic tremor'.

The anti-tremor effect of propranolol may prove to be specific, on the one hand, for essential tremor and, on the other, for the tremors produced by anxiety, thyrotoxicosis, or the administration of adrenaline or isoproterenol. Certainly, to date, we have not found propranolol to be effective in reducing the amplitude of any of the other types of tremor tested, including the underlying physiological tremor present in all normal individuals. This does not imply that the mechanisms for the production of all these propranolol-sensitive tremors are the same. Certainly their frequencies, electrophysiological patterns, and the time course of their response to various pharmacological agents differ, suggesting that the basic pathophysiology is not identical (Young et al., 1975; Growdon et al., 1975). Nevertheless, the response of a tremor to propranolol, plus its clinical and electrophysiological characteristics can enable us to recognize one or another of these common types of tremor in clinical situations where they might otherwise be overlooked, as in Parkinson's disease, or misdiagnosed, as in tremors associated with peripheral neuropathies. 
Using these criteria, it is possible to differentiate 'neuropathic tremor' from the tremor seen in some families with Charcot-Marie-Tooth disease (Shahani et al., 1973). This suggests that this latter type of tremor and the action tremor of Parkinson's disease are both simply essential tremor. In neither situation is it possible to distinguish them clinically from essential tremor, nor have we found any physiological or pharmacological differences.

Since DeJong (1926), observers, including Lance et al. (1963), have recognized an action tremor in Parkinson's disease which they differentiated from the tremor-at-rest; the latter authors emphasized the electromyographic differences between what they considered physiological tremor and the tremor-at-rest of Parkinson's disease. Our electrophysiological observations and the finding that the action tremor of Parkinson's disease is ameliorated by propranolol (Schwab and Young, 1971) suggest that certain patients with Parkinson's disease suffer from a clinically more significant essential tremor. On the basis of these findings, we suggest the term 'physiological tremor' be (1) restricted to the non-symptomatic tremor (in which no EMG bursts are obvious during multi-unit recordings) and (2) not used to describe propranolol-sensitive tremors (essential-familial-senile tremor or 'abnormal postural tremor'). Certainly, both Parkinson's disease and essential tremor are common, and the chance occurrence of both in a single patient would not be unexpected. The rather abrupt worsening of the long pre-existing essential tremor as the symptoms of Parkinson's disease begin suggests some pathophysiological mechanisms may be common to the two conditions. This would also be supported by the finding that stereotactic surgery, such as ventrolateral thalamotomy, can relieve contralateral essential tremor and the tremor-at-rest of Parkinson's disease, but is not useful in treating intention myoclonus or cerebellar ataxia ('tremor'). In any case, we have no evidence that these action tremors are in any way different from pure essential tremor.

If propranolol has a fairly specific action on essential tremor, by what mechanisms might it act? It has always been assumed that the pathophysiology underlying essential tremor would eventually be found to result from a derangement of the central nervous system at some level from spinal cord rostral. Observations that thalamotomy is effective in essential tremor and that it persists after deafferentation by ischaemia or procaine blocks support the assumption which is, of course, altogether reasonable. Certainly, propranolol does cross the blood-brain-barrier in animals and, presumably, in man. L-dopa increases the levels of central catecholamines and also worsens the action tremor of Parkinson's disease which, in turn, is reduced by propranolol. If this action of L-dopa is a central one, propranolol might act antagonistically at the same sites, but the status of central $\alpha$-and $\beta$-adrenergic receptors and their activity has not yet been clarified sufficiently so that one can be certain of the implications of the action of propranolol in terms of central neurophysiology (Young et al., 1975).

The mechanisms underlying action tremor 7 have been speculated upon in detail, but no $N$ convincing data are at hand. Neither do oup 옥 observations of the surface EMG characteristics of the various tremors shed light on the underlying mechanisms. However, the existence of neuropathic tremors (Adams et al., 1972) raise the question of possible peripheral factor responsible for tremor. Selective denervation and dysfunction of certain elements of the peripheral receptors, and/or servo loop, might produce quite different results from total deafferentation, as with dorsal rhizotomy. Therefore, the evidence that certain tremors persist after section of dorsal roots and that other subjects, in the same circumstances, do not develop tremor cannot be taken to prove the proposition that peripheral structures have no role to play in tremor. There is convincing evidence for peripheral $\beta$-adrenergic receptors concerned with tremor in normal subjects (Marsden et al., 1967; Young et al., 1975) and exaggeration of essential tremor in patients (Young et al., 1975). The elegant demonstration of what appear to be adrenergic endings on certain intrafusal fibres of the dog's muscle spindle by Banker and Girvin (1971) suggests that fusimotor and muscle afferent activity may be, in some way, related to the peripheral tremorgenic receptors described above. Though the site of these receptors remains to be proven, such findings, if forthcoming, would add 
considerably to our present meagre knowledge of tremor mechanisms.

\section{REFERENCES}

Adams, R. D., and Foley, J. M. (1953). Disorder of movement in more common varieties of liver disease. Electroencephalography and Clinical Neurophysiology, suppl. 3, 51 .

Adams, R. D., Shahani, B. T., and Young, R. R. (1972). Tremor in association with polyneuropathy. Transactions of the American Neurological Association, 97, $44-48$.

Banker, B. Q., and Girvin, J. P. (1971). The ultrastructural features of the mammalian muscle spindle. Journal of Neuropathology and Experimental Neurology, 30, 155-195.

Brumlik, J., and Yap, C. B. (1970). Normal Tremor. Thomas: Springfield, Ill.

Critchley, E. (1972). Clinical manifestations of essential tremor. Journal of Neurology, Neurosurgery, and Psychiatry, 35, 365-372.

Critchley, M. (1949). Observations on essential (heredofamilial) tremor. Brain, 72, 113-139.

De Jong, H. (1926). Action-tremor. Journal of Nervous and Mental Diseases, 64, 1-11.

Dupont, E., Hansen, H. J., and Dalby, M. A. (1973). Treatment of benign essential tremor with propranolol. Acta Neurologica Scandinavica, 49, 75-84.

Fahn, S. (1972). Differential diagnosis of tremors. Medical Clinics of North America, 56, 1363-1375.

Gilligan, B. S., Veale, J. L., and Wodak, J. (1972). Propranolol in the treatment of tremor. Practitioner, 207, 677-678.

Growdon, J. H., Shahani, B. T., and Young, R. R. (1975) The effect of alcohol on essential tremor. Neurology (Minneap.), 25, 259-262.

Hardy, J., Molina-Negro, P., Bertrand, C., and Martinez, N. S. (1970). Tremblement d'attitude. Union Médicale du Canada, 99, 449-456.

Hoefer, P. F. A., and Putnam, T. J. (1940). Action potentials of muscles in rigidity and tremor. Archives of Neurology and Psychiatry (Chic.), 43, 704-725.

Lance, J. W., Schwab, R. S., and Peterson, E. A. (1963). Action tremor and the cogwheel phenomenon in Parkinson's disease. Brain, 86, 95-110.
Leavitt, S., and Tyler, H. R. (1964). Studies in asterixis. Archives of Neurology (Chic.), 10, 360-368

Lippold, O. C. J., Redfearn, J. W. T., and Vuco, J. (1957). The rhythmical activity of groups of motor units in the voluntary contraction of muscle. Journal of Physiology (London), 137, 473-487.

Marsden, C. D., Foley, T. H., Owen, D. A. L. and McAllister, R. G. (1967). Peripheral $\beta$-adrenergic receptors concerned with tremor. Clinical Science, 33, 53-65.

Morgan, M. H., Hewer, R. L., and Cooper, R. (1973). Effect of the beta-adrenergic blocking agent propranolol on essential tremor.Journal of Neurology, Neurosurgery, and Psychiatry, 36, 618-624.

Murray, T. J. (1972). Treatment of essential tremor with propranolol. Canadian Medical Association Journal, 107, 984-986.

Pakkenberg, H. (1972). Propranolol in essential tremor. Lancet, 1, 633.

Schwab, R. S., and Young, R. R. (1971). Non-resting tremor in Parkinson's disease. Transactions of the American Neurological Association, 96, 305-307.

Sevitt, I. (1971). The effect of adrenergic beta-receptor blocking drugs on tremor. Practitioner, 207, 677-678.

Shahani, B. T., and Young, R. R. (1973). Some observations on tremor and other movement disorders. Electroencephalography and Clinical Neurophysiology, 34, 800 .

Shahani, B. T., Young, R. R., and Adams, R. D. (1973). The tremor in Roussy-Levy Syndrome. Neurology (Minneap.), 23, 425-426.

Tolosa, E. S., and Lowenson, R. B. (1975). Essential tremor: Treatment with propranolol. Neurology (Minneap.), 25, 1041-1044.

Winkler, G. F., and Young, R. R. (1971). The control of essential tremor by propranolol. Transactions of the American Neurological Association, 96, 66-68.

Winkler, G. F., and Young, R. R. (1974). Efficacy of chronic propranolol therapy in action tremors of the familial, senile, or essential varieties. New England Journal of Medicine, 290, 984-988.

Young, R. R., Growdon, J. H., and Shahani, B. T. (1975). Beta-adrenergic mechanisms in action tremor. New England Journal of Medicine, 293, 950-953.

Yudell, A., Dyck, P. J., and Lambert, E. H. (1965). A kinship with the Roussy-Levy syndrome. Archives of Neurology (Chic.), 13, 432-440. 